\title{
Brachio-cervical inflammatory myopathy associated with systemic sclerosis. Case series and review of literature
}

\author{
C.S.R. Araujo', R. Miossi' ${ }^{1}$, F.H.C. de Souza ${ }^{1}$, M.D. Costa ${ }^{2}$, A.M.S. da Silva ${ }^{3}$, \\ E.D. Campos ${ }^{3}$, E. Zanoteli ${ }^{3}$, S.K. Shinjo ${ }^{1}$ \\ ${ }^{1}$ Division of Rheumatology, Faculdade de Medicina FMUSP, Universidade de Sao Paulo, SP, Brazil; \\ ${ }^{2}$ Hospital Edmundo Vasconcelos, SP, Brazil; \\ ${ }^{3}$ Department of Neurology, Faculdade de Medicina FMUSP, Universidade de Sao Paulo, SP, Brazil
}

\section{SUMMARY}

This study was aimed at describing a case series of brachio-cervical inflammatory myopathy (BCIM) associated with systemic sclerosis (SSc), due to its rarity and limited coverage in published data. Another aim was to provide a literature review.

We reported four cases of BCIM-SSc from our tertiary center. In addition, we researched the literature and found six articles featuring 17 patients who fit this phenotype. We pooled all cases and reported their features. Most patients were female and had limited SSc, and the median time of BCIM presentation was three years after SSc diagnosis. Asymmetric muscle involvement, scapular winging, dropped head, axial weakness, camptocormia, dysphagia, and dermatomyositis stigmas were common features. All patients had esophageal involvement. Most had positive antinuclear antibody results, a scleroderma pattern in their capillaroscopy images, elevated serum creatine phosphokinase, myopathic electrophysiology, and muscle involvement in magnetic resonance imaging. Muscle histopathological findings varied widely, but in general all showed the presence of lymphoid infiltrates, muscle atrophy, increased MHC-I expression, MAC deposits, vasculopathy, and muscle fiber necrosis. The response to immunosuppressive therapy was highly irregular.

BCIM-SSc is a rare disorder that shares many similar phenotypes among the described cases, but has a highly heterogeneous response to treatment. At present, more data on the physiopathology, clinical features, and treatment is still needed.

Key words: Autoimmune diseases, inflammatory myopathies, myopathies, myositis, systemic sclerosis.

Reumatismo, 2021; 73 (2): 122-130

\section{INTRODUCTION}

$\mathrm{M}$ yopathies in systemic sclerosis (SSc) have been widely reported with a prevalence ranging from $13 \%$ to $81 \%(1-4)$. The muscular manifestations can vary from simple symptoms (e.g., myalgia, muscle fatigue) to severe and complex ones (e.g., proximal muscle weakness, dysphagia, and increased serum creatine phosphokinase) (1-4). The diagnosis of myopathy-associated SSc is still challenging due to the lack of specific criteria for defining these muscular manifestations in SSc (2).

Pestronk et al. (5) described 10 patients with a clinical variant of an inflammatory myopathy denominated brachio-cervical inflammatory myopathy (BCIM). The distinguishing features of BCIM are the prominent weakness of the proximal upper-limb and cervical muscles, as well as its association with other autoimmune diseases. In this case series (5), BCIM was associated with systemic lupus erythematosus, mixed connective tissue disease, Sjögren's disease, rheumatoid arthritis, myasthenia gravis, and diabetes mellitus. Histopathological changes in muscle biopsy included variation in the sizes of the muscle fibers, necrosis, major histological compatibility (MHC)-I expression in muscle fibers, membrane attack complex (MAC) deposits, mononuclear (mainly CD20+ and CD4+ lymphocytes) and macrophage infil- 
trates, and focal accumulations of mononuclear cells at the perimysium and perivascular sites (5).

However, BCIM associated with SSc (BCIM-SSc) has been scarcely described in the literature (6-11). Therefore, we aimed to report a series of four patients with BCIM-SSc from our tertiary center. In addition, we reviewed the literature on this association.

\section{PATIENTS AND METHODS}

This was a retrospective study to evaluate a cohort of four patients with BCIM-SSc followed at our tertiary center from 2017 to 2020. The study was approved by the local ethics committee.

All patients fulfilled the 2013 ACR/EULAR classification criteria for SSc (12) and the 2017 EULAR/ACR classification criteria for inflammatory myopathies (13). We considered the diagnosis of BCIM when patients presented with an inflammatory myopathy with predominance of upper limb and cervical weakness in a physical examination score such as Medical Resource Council (MRC) or Muscle Manual Testing (MMT-8).

Literature review. We conducted literature searches on electronic databases (Medline/ PubMed). The selection of the descriptors was carried out in September 2020, based on the following keywords: "systemic sclerosis", "scleroderma", "brachio-cervical inflammatory myopathy", "brachio-cervical myositis", "scleromyositis", "dropped head syndrome", "scapular myopathy". The keywords were combined with the Boolean operators of "OR" and "AND" and they were adapted for each database as required. In addition, the reference list of each retrieved article was reviewed manually. The following inclusion criteria were adopted: no chronological limit, original articles, case reports, and case series. We excluded abstracts of conferences, monographies, theses and dissertations, articles related to other myopathies and letters to the Editor with commentaries from the review. The search led to the retrieval of 747 articles with case reports. Of these, six con- tained cases of SSc and an inflammatory myopathies that fit the BCIM phenotype (6-11). The main features of these patients are shown in the Tables I-III.

\section{CASE REPORTS}

\section{Patient \#1}

A 40-year-old woman with a five-year history of limited SSc presented with asymmetrical proximal muscle weakness in the arms and legs with MRC and MMT-8 scores of 48 and 62 , respectively, as well as elevated serum concentration of creatine phosphokinase (CPK). Antinuclear antibody test (ANA) was positive with a nucleolar pattern and a 1:80 titer; anti-DNA, anti-RNP, anti-Smith, anti-Ro, anti-La, anti-SCL70, anti-Jo1 and rheumatoid factor (RF) were all negative. Magnetic resonance imaging (MRI) of the thighs showed diffuse muscle edema. In addition, nerve conduction studies were normal and electromyography (EMG) showed a myopathic pattern in the upper limbs and cervical girdle. A muscle biopsy of the right biceps revealed intense inflammation, lymphoid follicles, and a dystrophic pattern. The patient received $2 \mathrm{~g} / \mathrm{kg}$ of intravenous human immunoglobulin (IVIg) and methotrexate, which led to initial improvement of symptoms and CPK decrease (maximum of 3387 $\mathrm{U} / \mathrm{L})$. After one year of therapy, the disease flared up with worsening of shoulder and neck weakness (MRC scale of 48 in the deltoids and neck flexors), CPK increase, and muscle edema, atrophy, and fatty infiltration at MRI (Figure 1A-B). In addition to IVIg and methotrexate, mycophenolate mofetil was initiated, but the disease continued to worsen.

\section{Patient \#2}

A 35-year-old woman presented with an eight-year history of limited SSc, as well as a seven-year history of weakness in the proximal lower limbs and proximal upper and cervical muscles (MRC sum score of 48 and MMT8 score of 62), dysphagia, heliotrope rash, Gottron's papules, interstitial lung disease, and elevated serum levels of CPK (maximum of $2782 \mathrm{U} / \mathrm{L}$ ). ANA was 
positive with a nucleolar pattern and a 1:640 titer; anti-DNA, anti-RNP, anti-Smith, antiRo, anti-La, anti-SCL70 and anti-Jo1 were negative. MRI of the cervical and pelvic girdles showed muscle edema, atrophy, and fatty infiltration. Prescribed medications included prednisone, methotrexate, azathioprine, mycophenolate mofetil, and IVIg $(2 \mathrm{~g} / \mathrm{kg})$ which resulted in adequate control of SSc activity with improvement of Rodnan skin score, but poor response of myositis, with a modest decrease of MRC sum score to 44 and MMT-8 score to 56.
Recently, she was started on rituximab (2 grams every 6 months) which led to an improvement in serum CPK level but not in muscle strength.

\section{Patient \#3}

A 31-year-old woman presented with a fiveyear history of limited SSc, as well as a fouryear history of symmetrical proximal muscle weakness in the upper limbs, with deltoid strength of III and neck flexor strength of II in the MRC scale. Her MRC sum score was 48 and MMT-8 score was 56. ANA was

Table I - General features of the patients from the present study and the literature.

\begin{tabular}{|c|c|c|c|c|c|c|c|c|}
\hline & $\mathrm{N}^{\circ}$ & Sex & Age & $\begin{array}{c}\text { Age at } \\
\text { myopathy onset }\end{array}$ & $\begin{array}{c}\text { Age at SSc } \\
\text { onset }\end{array}$ & $\begin{array}{c}\text { Muscle weakness } \\
\text { at onset }\end{array}$ & Other clinical features & SSc type \\
\hline \multirow{4}{*}{ Present study } & 1 & $\mathrm{~F}$ & 40 & 36 & 34 & $\begin{array}{c}\text { Cervical, prox UL, } \\
\text { prox LL }\end{array}$ & $\begin{array}{l}\text { Asymmetry, scapular } \\
\text { winging }\end{array}$ & Limited \\
\hline & 2 & $\mathrm{~F}$ & 39 & 28 & 27 & $\begin{array}{l}\text { Cervical, prox UL, } \\
\text { prox LL }\end{array}$ & $\begin{array}{l}\text { Heliotrope, Gottron's } \\
\text { papules, dysphagia }\end{array}$ & Limited \\
\hline & 3 & $\mathrm{~F}$ & 32 & 27 & 26 & Cervical, prox UL & Dysphagia & Limited \\
\hline & 4 & $\mathrm{~F}$ & 43 & 42 & 38 & Cervical, prox UL & $\begin{array}{l}\text { SCT } 6 \text { months prior } \\
\text { to myopathy onset, } \\
\text { deltoid atrophy }\end{array}$ & Diffuse \\
\hline \multirow{8}{*}{$\begin{array}{l}\text { Suarez- } \\
\text { Calvet } \\
\text { et al. (11) }\end{array}$} & 1 & $\mathrm{~F}$ & & 57 & 20 & Cervical, prox UL & Asymmetry & \\
\hline & 2 & $\mathrm{~F}$ & & 75 & 00 & Cervical, prox UL & Axial weakness & \\
\hline & 3 & $\mathrm{~F}$ & & 39 & +2 & Cervical, prox UL & Scapular winging & \\
\hline & 4 & $\mathrm{~F}$ & & 61 & 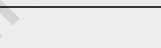 & Cervical, prox UL & Distal UL, axial weakness & \\
\hline & 5 & $\mathrm{~F}$ & & 26 & & Cervical, prox UL & Scapular winging & \\
\hline & 6 & $\mathrm{~F}$ & & 66 & & Cervical, prox UL & Scapular winging & \\
\hline & 7 & $\mathrm{~F}$ & & 47 & & Cervical, prox UL & & \\
\hline & 8 & $\mathrm{~F}$ & & 33 & & Cervical, prox UL & & \\
\hline $\begin{array}{l}\text { Gao } \\
\text { et al. (10) }\end{array}$ & 1 & $F$ & & 31 & 31 & Cervical, prox UL & Asymmetry, dysphagia & Limited \\
\hline $\begin{array}{l}\text { Yoshida } \\
\text { et al. (8) }\end{array}$ & 1 & $\mathrm{~F}$ & 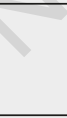 & 66 & 66 & Cervical, prox UL & $\begin{array}{l}\text { Axial, dropped head, } \\
\text { camptocormia, } \\
\text { dysphagia, dysarthria }\end{array}$ & Limited \\
\hline $\begin{array}{l}\text { Fernández- } \\
\text { Serna } \\
\text { et al. (7) }\end{array}$ & 1 & M & & 51 & 51 & Cervical & $\begin{array}{l}\text { Dropped head, Gottron's } \\
\text { papules, heliotrope }\end{array}$ & Diffuse \\
\hline $\begin{array}{l}\text { Garcin } \\
\text { et al. (6) }\end{array}$ & 1 & $F$ & & 50 & & $\begin{array}{l}\text { Cervical, prox UL, } \\
\text { prox LL }\end{array}$ & $\begin{array}{c}\text { Dropped head, } \\
\text { dysphagia }\end{array}$ & \\
\hline \multirow{5}{*}{$\begin{array}{l}\text { Rojana- } \\
\text { Udomsart } \\
\text { et al. (9) }\end{array}$} & 1 & $\mathrm{~F}$ & & 27 & 25 & $\begin{array}{l}\text { Cervical, prox UL, } \\
\text { prox LL }\end{array}$ & Calcinosis & Limited \\
\hline & 2 & $\mathrm{~F}$ & & 43 & 38 & $\begin{array}{l}\text { Cervical, prox UL, } \\
\text { prox LL }\end{array}$ & $\begin{array}{c}\text { History of } \\
\text { D-penicilamine }\end{array}$ & Diffuse \\
\hline & 3 & $M$ & & 51 & 50 & $\begin{array}{l}\text { Cervical, prox UL, } \\
\text { prox LL }\end{array}$ & $\begin{array}{c}\text { History of } \\
\text { D-penicilamine }\end{array}$ & Localized \\
\hline & 4 & $\mathrm{~F}$ & & 53 & 49 & $\begin{array}{l}\text { Cervical, prox UL, } \\
\text { prox LL }\end{array}$ & $\begin{array}{l}\text { Axial weakness, } \\
\text { camptocormia }\end{array}$ & Limited \\
\hline & 5 & $M$ & & 52 & 49 & $\begin{array}{l}\text { Cervical, prox UL, } \\
\text { prox LL }\end{array}$ & $\begin{array}{l}\text { Axial weakness, } \\
\text { camptocormia }\end{array}$ & Diffuse \\
\hline
\end{tabular}

F, female; LL, lower limbs; M, male; Prox, proximal; SSc, systemic sclerosis; SCT, stem cell transplant; UL, upper limbs. 
positive with a nucleolar pattern and a 1:160 titer; anti-DNA, anti-RNP, anti-Smith, antiRo, anti-La, anti-SCL70 and anti-Jo1 were negative. MRI showed edema of the shoul- der muscles. In addition, CPK was slightly elevated (maximum of $303 \mathrm{U} / \mathrm{L}$ ), EMG was suggestive of myopathy with normal nerve conduction studies, and muscle biopsy

Table II - General exams of the patients from the present study and the literature.

\begin{tabular}{|c|c|c|c|c|c|c|c|c|c|}
\hline & $\mathbf{N}^{\circ}$ & 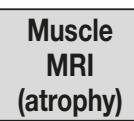 & $\begin{array}{l}\text { Esophageal } \\
\text { manometry }\end{array}$ & $\begin{array}{l}\text { Lung } \\
\text { disease } \\
\text { CT }\end{array}$ & Spirometry & $\mathrm{CPU}$ & $\begin{array}{c}\text { Echo } \\
\text { cardiogram }\end{array}$ & EMG & Muscle biopsy \\
\hline \multirow{4}{*}{$\begin{array}{l}\text { Present } \\
\text { study }\end{array}$} & 1 & $\begin{array}{l}\text { Th, UL not } \\
\text { performed }\end{array}$ & & & $\begin{array}{l}\text { FEV1 82\% } \\
\text { FVC } 84 \% \\
\text { DLCO N/A }\end{array}$ & & Normal & Myopathic & LI, LF, atrophy \\
\hline & 2 & $\begin{array}{c}\mathrm{De} / \mathrm{Ps} / \mathrm{C} / \\
\mathrm{Th}\end{array}$ & & Yes & $\begin{array}{l}\text { FEV1 59\% } \\
\text { FVC 58\%, } \\
\text { DLCO 47\% }\end{array}$ & SD & Normal & Normal & \\
\hline & 3 & $\mathrm{De} / \mathrm{Ps} / \mathrm{Th}$ & & Yes & $\begin{array}{l}\text { FEV1 98\% } \\
\text { FVC 93\% } \\
\text { DLCO N/A }\end{array}$ & SD & Normal & Myopathic & LF, MHC1 (+) \\
\hline & 4 & $\mathrm{De}$ & Last $1 / 3$ & & $\mathrm{~N} / \mathrm{A}$ & & 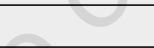 & Myopathic & LF \\
\hline \multirow{8}{*}{$\begin{array}{l}\text { Suarez- } \\
\text { Calvet } \\
\text { et al. (11) }\end{array}$} & 1 & $\mathrm{Ps} / \mathrm{De}$ & Last $1 / 3$ & & & SD & Normal & & \\
\hline & 2 & Ps & Last $1 / 3$ & Yes & & SD & Normal & & \\
\hline & 3 & $\mathrm{De} / \mathrm{C}$ & total & Yes & & SD & Normal & & \\
\hline & 4 & $\mathrm{Ps} / \mathrm{C}$ & total & & & SD & Normal & & \\
\hline & 5 & $\mathrm{Ps} / \mathrm{De}$ & Last $1 / 3$ & Yes & +2 & SD & Normal & & \\
\hline & 6 & $C$ & & & $C$ & SD & Normal & & \\
\hline & 7 & $\mathrm{C}$ & Last $1 / 3$ & & 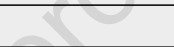 & SD & Normal & & \\
\hline & 8 & $\mathrm{C}$ & Last $1 / 3$ & Yes & 82 & SD & Normal & & \\
\hline $\begin{array}{l}\text { Gao } \\
\text { et al. (10) }\end{array}$ & 1 & Normal & & & Unremarkable & SD & & Myopathic & $\begin{array}{l}\text { LI (B-cells), LF, } \\
\text { MAC depositions }\end{array}$ \\
\hline $\begin{array}{l}\text { Yoshida } \\
\text { et al. (8) }\end{array}$ & 1 & $\mathrm{C} / \mathrm{UL}$ & & Yes & CVF $33.5 \%$ & SD & & Myopathic & $\begin{array}{l}\text { FSD, atrophy, } \\
\text { necrosis, rimmed } \\
\text { vacuoles }\end{array}$ \\
\hline $\begin{array}{l}\text { Fernández- } \\
\text { Serna } \\
\text { et al. (7) }\end{array}$ & 1 & Normal & & & Restrictive & SD & Normal & Myopathic & Normal \\
\hline $\begin{array}{l}\text { Garcin } \\
\text { et al. (6) }\end{array}$ & 1 & Normal & $\Delta$ & Yes & & & & Myopathic & $\begin{array}{l}\text { Necrosis, LI in } \\
\text { endo, peri and } \\
\text { epimyosium }\end{array}$ \\
\hline \multirow{5}{*}{$\begin{array}{l}\text { Rojana- } \\
\text { Udomsart } \\
\text { et al. (9) }\end{array}$} & 1 & & & & & & & Myopathic & $\begin{array}{c}\text { NIM, scleroderma } \\
\text { vasculopathy }\end{array}$ \\
\hline & 2 & & & Yes & & & & Myopathic & $\begin{array}{l}\text { NIM, scleroderma } \\
\text { vasculopathy }\end{array}$ \\
\hline & 3 & & & & & & & Myopathic & $\begin{array}{l}\text { NIM, scleroderma } \\
\text { vasculopathy }\end{array}$ \\
\hline & 4 & & & & & & & Myopathic & $\begin{array}{l}\text { Focal interstitial } \\
\text { inflammation, } \\
\mathrm{MHCl}(+) \text {, atrophy } \\
\text { and fibrosis, } \\
\text { scleroderma } \\
\text { vasculopathy }\end{array}$ \\
\hline & 5 & & & & & & & & $\begin{array}{c}\mathrm{Fl}, \mathrm{MHCl} \\
\text { scleroderma } \\
\text { vasculopathy }\end{array}$ \\
\hline
\end{tabular}

C, cervical; CPU, capillaroscopy; CT, computed tomography; De, deltoid; FEV, forced vital capacity; FVC, forced vital capacity; FSV, fiber size variation; LF, lymphoid follicles; LI, lymphocyte infiltrate; MHC, major histocompatibility complex; MRI, magnetic resonance images; N/A, not available; NIM, necrotizing inflammatory myopathies; Ps, periscapular; SD, sclera; Th, thighs; UL, upper limbs. 
showed intense inflammation, lymphoid follicles, and a dystrophic pattern (Figure 2AE). She has received $2 \mathrm{~g} / \mathrm{kg}$ of IVIg, methylprednisolone pulse therapies, prednisone, methotrexate with mycophenolate mofetil, and rituximab with no response, and her muscle strength continues to deteriorate (last MRC of 42 and MMT8 of 50).

Table III - General laboratory examinations, treatment and disease status outcome of the patients from the present study and the literature.

\begin{tabular}{|c|c|c|c|c|c|c|c|}
\hline & $\mathbf{N}^{0}$ & ANA & Others & HLA & $\begin{array}{l}\text { Maximum } \\
\text { CPK (U/L) }\end{array}$ & Treatment & Outcome \\
\hline \multirow{4}{*}{$\begin{array}{l}\text { Present } \\
\text { study }\end{array}$} & 1 & $\begin{array}{l}\text { Nucleolar } \\
1 / 80\end{array}$ & - & & 3387 & $\begin{array}{l}\text { Pred, IVIg, MTX, } \\
\text { MMF, RTX }\end{array}$ & $\begin{array}{c}\text { Response to Pred/MTX/ } \\
\text { IVlg }\end{array}$ \\
\hline & 2 & $\begin{array}{l}\text { Nucleolar } \\
1 / 640\end{array}$ & - & & 2782 & $\begin{array}{l}\text { Pred, MTX, MMF, } \\
\text { IVIg, RTX }\end{array}$ & $\begin{array}{c}\text { Worsening, stable } \\
\text { myopathy and CPK } \\
\text { improvement after RTX }\end{array}$ \\
\hline & 3 & $\begin{array}{c}\text { Nucleolar } \\
1 / 160\end{array}$ & - & & 303 & $\begin{array}{l}\text { Pred, IVIg, MTX, } \\
\text { MMF, RTX }\end{array}$ & Worsening \\
\hline & 4 & $\begin{array}{c}\text { Nucleolar } \\
1 / 1280\end{array}$ & - & & 35 & Pred, IVIg, MTX & Stability \\
\hline \multirow{8}{*}{$\begin{array}{l}\text { Suarez- } \\
\text { Calvet } \\
\text { et al. (11) }\end{array}$} & 1 & $\begin{array}{c}\text { Nucleolar } \\
1 / 640\end{array}$ & - & $\begin{array}{l}\text { DRB1*03:01 } \\
\text { DRB1*11:02 }\end{array}$ & & Pred, IVIg, MMF & Stability \\
\hline & 2 & $\begin{array}{c}\text { Nucleolar } \\
1 / 640\end{array}$ & $\mathrm{Ku}$ & $\begin{array}{l}\text { DRB1*03:02 } \\
\text { DRB1*04:02 }\end{array}$ & & Pred, MTX & Improved \\
\hline & 3 & $\begin{array}{l}\text { Speckled } \\
1 / 80\end{array}$ & - & $\begin{array}{l}\text { DRB1*03:01 } \\
\text { DRB1*04:02 }\end{array}$ & & $\begin{array}{l}\text { Pred, IVIg, MMF, } \\
\text { MTX, CP, CYC }\end{array}$ & Stability \\
\hline & 4 & $\begin{array}{c}\text { Nucleolar } \\
1 / 160\end{array}$ & Th/To & & & $\begin{array}{l}\text { Pred, AZA, MTX, } \\
\text { MMF }\end{array}$ & Worsening, death \\
\hline & 5 & $\begin{array}{c}\text { Speckled } \\
1 / 320\end{array}$ & $\mathrm{Ku}$ & $\begin{array}{l}\text { DRB1*13:01 } \\
\text { DRB1*15:01 }\end{array}$ & & Pred, MMF & Improved \\
\hline & 6 & Negative & & $\begin{array}{l}\text { DRB1*11:02 } \\
\text { DRB1 }^{*} 03: 01\end{array}$ & & Pred, MMF & Stability \\
\hline & 7 & $\begin{array}{l}\text { Speckled } \\
1 / 80 \\
\end{array}$ & & $\begin{array}{l}\text { DRB1*04:05 } \\
\text { DRB1*13:01 }\end{array}$ & & Pred, MMF & Improved \\
\hline & 8 & $\begin{array}{c}\text { Speckled } \\
1 / 1280\end{array}$ & $\begin{array}{l}\text { Ro52, } \\
\text { Gp210 }\end{array}$ & $\begin{array}{l}\text { DRB1*03:01 } \\
\text { DRB1*11:04 }\end{array}$ & & Pred, IVlg, MMF & Improved \\
\hline $\begin{array}{l}\text { Gao } \\
\text { et al. (10) }\end{array}$ & 1 & $\begin{array}{l}\text { Positive (no pattern } \\
\text { or titre given) }\end{array}$ & $\begin{array}{l}\text { Ro52, Ku, } \\
\text { dsDNA }\end{array}$ & & 5085 & Pred, AZA, IVlg & Improved \\
\hline $\begin{array}{l}\text { Yoshida } \\
\text { et al. (8) }\end{array}$ & 1 & $\begin{array}{c}\text { Homogenous, } \\
\text { speckled, nucleolar } \\
1 / 640\end{array}$ & $\mathrm{Ku}$ & & 4122 & $\begin{array}{c}\text { Pred, IVIg, } \\
\text { Tacrolimus, AZA }\end{array}$ & Improved \\
\hline $\begin{array}{l}\text { Fernández- } \\
\text { Serna } \\
\text { et al. (7) }\end{array}$ & 1 & $\begin{array}{l}\text { Nucleolar } \\
1 / 1280\end{array}$ & $\mathrm{PM} / \mathrm{Scl}$ & & 232 & Pred, MTX & Improved \\
\hline $\begin{array}{l}\text { Garcin } \\
\text { et al. (6) }\end{array}$ & 1 & $\begin{array}{c}1 / 640 \\
\text { (no pattern given) }\end{array}$ & $\mathrm{PM} / \mathrm{Scl}$ & & 1224 & Pred & Improved \\
\hline \multirow{5}{*}{$\begin{array}{l}\text { Rojana- } \\
\text { Udomsart } \\
\text { et al. (9) }\end{array}$} & 1 & Nucleolar & $\begin{array}{l}\text { Scl70,ENA, } \\
\text { Jo-1 }\end{array}$ & & 1260 & Pred, MTX, CYC & Worsening \\
\hline & 2 & Nucleolar & - & & 619 & Pred, MTX & $\begin{array}{l}\text { Improved, worsened after } \\
\text { MTX was removed }\end{array}$ \\
\hline & 3 & Speckled & - & & 400 & Pred, MTX, IVlg & Improved \\
\hline & 4 & Negative & - & & 287 & - & Worsening \\
\hline & 5 & $\begin{array}{l}\text { Speckled } \\
1 / 640\end{array}$ & - & & 806 & Pred, MTX, AZA & Worsening \\
\hline
\end{tabular}

ANA, antinuclear antibody; Aza, azathioprine; CP, cyclosporine; CYC, cyclophosphamide; HLA, human leukocyte antigen; IVlg, intravenous human immunoglobulin; MMF, mycophenolate mofetil; MTX, methotrexate; Pred, prednisone. 


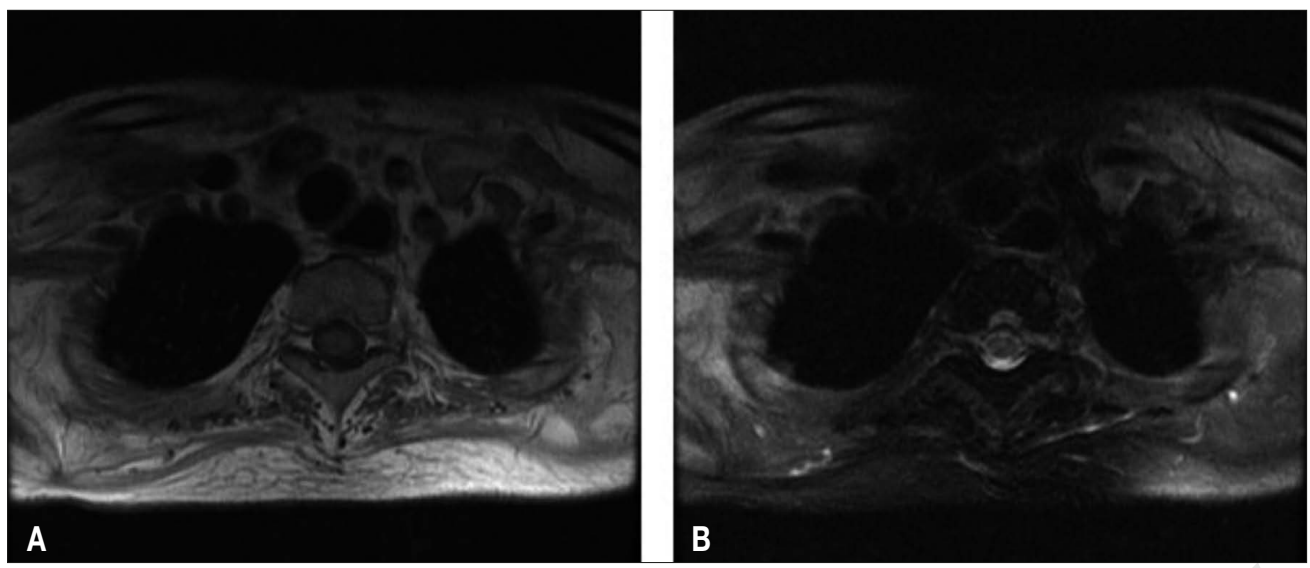

Figure 1 - MRI of the cervical girdle of patient \#1. A) T1-weighted sequence showing bilateral paraspinal and periscapular muscle atrophy and fatty replacement. B) T2-weighted sequence with fat saturation showing muscle edema of the right periscapular muscles.

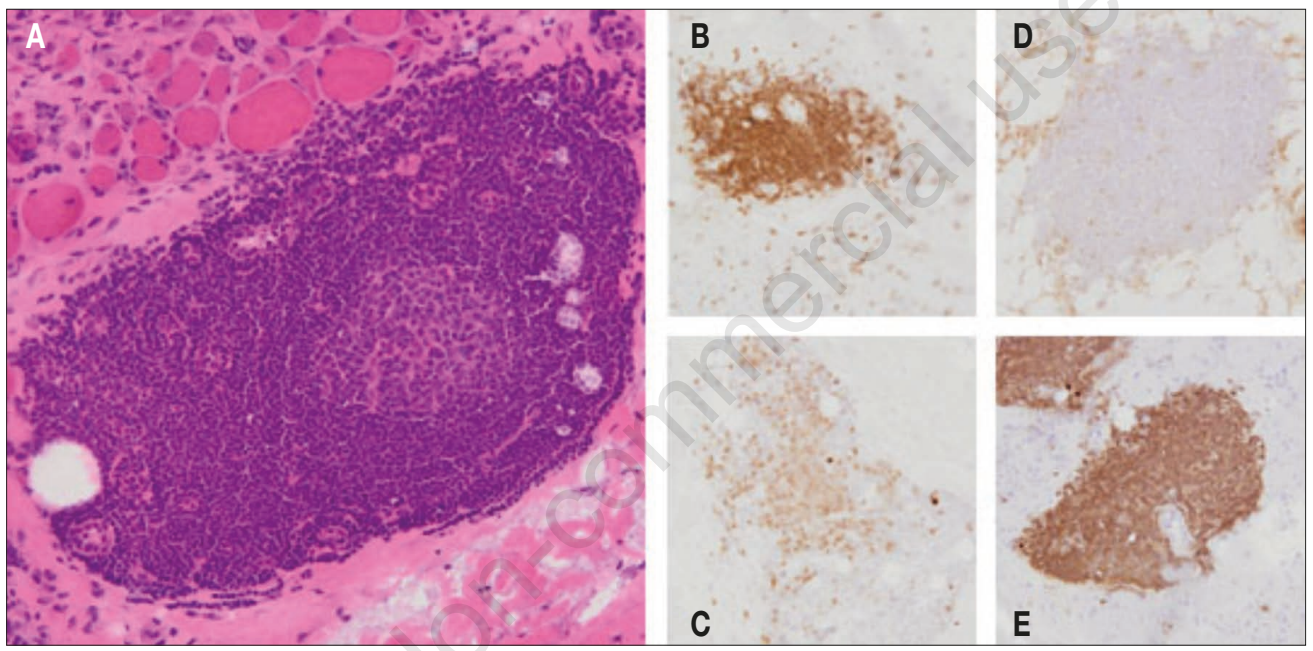

Figure 2 - Muscle biopsy from the patient \#3. There is an intense inflammation resembling lymphoid follicle pattern on muscle biopsy. Stain: A) hematoxilin-eosin; B) CD4; C) CD8; D) CD68; and E) CD20.

\section{Patient \#4}

A 42-year-old woman presented with a four-year history of diffuse SSc with pulmonary hypertension and severe skin involvement, which greatly limited her movement. She underwent an autologous hematopoietic stem cell transplant at the age of 40 as treatment for SSc, with great improvement in the skin condition. After six months, she developed muscle weakness, especially for arm abduction, and she noticed progressive atrophy in the deltoid muscles. Her initial MRC sum score was 54 and MMT8 score was 66. ANA was posi- tive with a nucleolar pattern and a 1:1280 titer; anti-DNA, anti-RNP, anti-Smith, antiRo, anti-La, anti-SCL70 and anti-Jo1 were negative. Serum CPK levels were normal, and EMG showed a myopathic pattern with normal nerve conduction studies. Deltoid muscle biopsy showed lymphoid follicles associated with a dystrophic pattern. She was started on prednisone, IVIg ( $2 \mathrm{~g} / \mathrm{kg})$, and methotrexate, and the disease stabilized.

Additional information on these four patients, and data collected from literature review are presented in Tables I-III. 


\section{DISCUSSION AND CONCLUSIONS}

In the present study, we described four patients with BCIM-SSc. All patients were female with a median of two years since SSc diagnosis and myopathy onset. Most patients had limited skin disease, and all had positive antinuclear antibodies. All of them had muscle edema in their MRI images and myopathic pattern on EMG. In these four cases, we noted that the usual immunosuppressive drugs did not lead to a satisfactory response.

Pestronk et al. (5) described the first cases of BCIM in 2006. Since then, six articles compounding 17 patients of BCIM specifically associated with SSc have been published (6-11). Chronologically, Garcin et al. (6) reported a first case of dropped head syndrome as scleromyositis suggestive of SSc and BCIM phenotype. A muscle biopsy contained changes similar to those of patients described in Pestronk et al. paper (5). Fernández-Serna et al. (7), and Yoshida et al. (8) also reported one case each of dropped head syndrome due to inflammatory myopathies (classified as scleromyositis and scleroderma-polymyositis overlap syndrome, respectively) concomitant with SSc onset. These reports seem to fit the BCIM phenotype and were added to this review. Rojana-Udomsart et al. (9) reported five patients with SSc and BCIM, of which three presented with dropped head syndrome and two with the inability to stand upright (camptocormia). The histopathological findings were heterogeneous, although all patients had scleroderma vasculopathy. Gao et al. (10) described one case of BCIM that actually preceded the onset of SSc, and muscle histopathology showed B cell infiltrates and MAC deposits. Finally, Suarez-Calvet et al. (11) reported eight cases of SSc and BCIM with thorough clinical, serological, and histopathological analyses. Their findings included an increased prevalence of HLA-DRB1*03:01 and HLA-DRB1*13:01, and they compared the histopathological changes in the muscle biopsies of these patients with healthy, dermatomyositis or immune-mediated necrotizing myopathy controls. BCIM patients had increased myofiber size variability, muscle atrophy, necrosis, MHC-I expression and CD68 macrophages infiltrates. Some biopsies showed CD20+, CD4+, and CD8+ infiltrates, as well as membrane attack complex (MAC) deposits. This study also showed increased serum levels of thrombospondin-1 (a cytokine involved in tissue response to hypoxia, extracellular matrix organization, and macrophage infiltration), which correlated with a more severe histopathological aspect of the myopathy (12-15).

In the present study, the pooled analysis showed common demographic and clinical features among the cases. For instance, 18 out of 21 patients were female. Therefore, our four patients corroborated the literature data $(2,4)$. The myopathic process initiates within the first few years of the diagnosis of SSc $(4,6-8,10,11)$. The median time of BCIM presentation was three years after SSc diagnosis (two years in our series). In addition, the pattern of SSc was limited in seven out of 12 patients, diffuse in four out of 12 patients, and localized in one patient (7-10). In our series, limited and diffuse disease phenotypes occurred in patients and one patient, respectively. Moreover, all cases had cervical weakness, and all but one had proximal upper limb weakness. Lower limb weakness was less common (eight out of 21) (6-11). Other clinical features included asymmetric muscle involvement, scapular winging, dropped head, axial weakness, camptocormia, dysphagia, and dermatomyositis features (heliotrope rash, Gottron's papules) (6-11). Interstitial lung disease was present in nine out of 17 cases $(6-8,10,11)$. All patients with esophagic manometry had esophageal involvement (11).

Regarding treatment, two patients had a prior history of D-penicillamine use (9), and one patient had a recent history of autologous stem cell transplant as treatment for SSc. The association of D-penicillamine and inflammatory myopathy has been well established (16-18). Regarding stem cell transplants, inflammatory myopathy has usually been described as a spec- 
trum of the graft versus host disease, and mostly in case of allogenic stem cell transplants (19-21).

Concerning laboratory parameters, 19 out of 21 cases had positive antinuclear antibodies, with speckled and nucleolar patterns being the most common (6-11). In our series, one patient had speckled and three patients had nucleolar patterns. The most common antibody found was anti-Ku (four out of 21 cases) $(8,10,11)$, although not all patients had a full antibody workup done. None of our patients had specific antibodies. The serum levels of CPK varied widely (35-5085 U/L) (6-11). In our series, one of the patients had a normal serum level of CPK

With regard to complementary exams, MRI were mostly positive ( 13 out of 16 cases) for edema or atrophy signs $(6-8,10,11)$. All of our cases had positive MRI image findings and the main findings were edema and fatty infiltration. Capillaroscopy showed a scleroderma pattern in 13 out of 14 patients ( 7 , $8,10,11)$. No abnormal echocardiograms were described. In addition, EMG showed myopathic patterns in 11 out of 12 patients (6-10). In the present study, only one of the patients had normal EMG.

Histopathological analysis varied widely between the case reports. A common thread among them was the presence of lymphoid infiltrates, muscle atrophy, increase of MHC-I expression, MAC deposits, vasculopathy, and muscle necrosis (6-11). Our cases showed lymphoid follicular structures, which were previously described in juvenile and adult dermatomyositis (22-24).

The response to immunosuppressive therapy was also highly variable (6-11). In our experience, we have found these patients to be challenging and refractory to treatment, although other reports showed a trend toward stability or improvement. The drugs used for treatment also varied greatly, although all treated patients received glucocorticoids. In other reports of inflammatory myopathy in SSc, elevated serum levels of CPK have been associated with a poor prognosis (25-27) and treatment usually included glucocorticoids and immunosuppressive drugs $(1,2,4)$. IVIg has been shown to be a glucocorticoid-sparing treatment (28), and histologic muscle inflammation has been described as a marker for a favorable treatment response in SScassociated myopathies (29).

A limitation of the present study is the heterogeneity of the data in the published reports. Not all participants reported clinical aspects, such as the classification of SSc. All patients had ANA tests, though data on titer and pattern was not always reported. Specific antibodies were not performed in all patients. Other diagnostic tests, such as echocardiogram, spirometry, and capillaroscopy, also were not always performed. All patients of Suarez-Calvet et al. (11) were HLA-typed. Histopathological data were challenging to pool because not all reports had the same analysis (e.g., testing for MHC-I, MAC complexes). BCIM is still not a widely known entity, and three out of the six articles (6-8) did not report the cases as BCIM.

In conclusion, BCIM-SSc is a rare condition, and its clinical features, epidemiology, and physiopathology are still not well known. The patients reported in this review tended to be female, have positive speckled or nucleolar antinuclear antibodies, scleroderma patterns by capillaroscopy, abnormal esophageal manometry results, and muscle inflammation or atrophy at MRI and EMG. This myopathy seems to happen within the first years of SSc. No histopathological finding was pathognomonic, but we believe that the presence of lymphoid follicle-like structures, especially with $B$ cell infiltrates, might be a specific finding for BCIM. Response to treatment was variable. At this time, more data on the physiopathology, clinical features, and treatment are needed.

\section{Funding}

This work was funded by Conselho $\mathrm{Na}$ cional de Desenvolvimento Científico e Tecnológico (CNPq) 303379/2018-9 and Faculdade de Medicina da USP to SKS.

\section{Conflict of interests}

All authors declare that they have no conflict of interests. 


\section{REFERENCES}

1. Paik JJ. Myopathy in scleroderma and in other connective tissue diseases. Curr Opin Rheumatol. 2016; 28: 631-635.

2. Paik JJ. Muscle disease in scleroderma. Curr Opin Rheumatol. 2018; 30: 576-580.

3. Mimura Y, Ihn H, Jinnin M, et al. Clinical and laboratory features of scleroderma patients developing skeletal myopathy. Clin Rheumatol. 2005; 24: 99-102.

4. Ranque B, Authier FJ, Berezne A, et al. Systemic sclerosis-associated myopathy. Ann N Y Acad Sci. 2007; 1108: 268-282.

5. Pestronk A, Kos K, Lopate G, Al-Lozi MT. Brachio-cervical inflammatory myopathies: Clinical, immune, and myopathologic features. Arthritis Rheum. 2006; 54: 1687-1696.

6. Garcin B, Lenglet T, Dubourg O, et al. Dropped head syndrome as a presenting sign of scleromyositis. J Neurol Sci. 2010; 292: 101-103.

7. Fernández-Serna M, Arboleya L, Alonso S, et al. Dropped head syndrome in a patient with scleromyositis. J Clin Rheumatol. 2013; 19: 32-34.

8. Yoshida T, Yoshida M, Mitsuyo K, et al. Dropped head syndrome and the presence of rimmed vacuoles in a muscle biopsy in scleroderma-polymyositis overlap syndrome associated with anti-ku antibody. Intern Med. 2018; 57: 887-891.

9. Rojana-Udomsart A, Fabian V, Hollingsworth $\mathrm{PN}$, et al. Paraspinal and scapular myopathy associated with scleroderma. J Clin Neuromuscul Dis. 2010; 11: 213-222.

10. Gao AF, Saleh PA, Kassardjian CD, et al. Brachio-cervical inflammatory myopathy with associated scleroderma phenotype and lupus serology. Neurol Neuroimmunol Neuroinflammation. 2018; 5: 1-3.

11. Suárez-Calvet X, Alonso-Pérez J, Castellví I, et al. Thrombospondin-1 mediates muscle damage in brachio-cervical inflammatory myopathy and systemic sclerosis. Neurol Neuroimmunol Neuroinflammation. 2020; 7: e694.

12. van den Hoogen F, Khanna D, Fransen J, et al. Classification criteria for systemic sclerosis: An American College of Rheumatology/European League Against Rheumatism Collaborative Initiative. Arthritis Rheum. 2013; 65: 2737-2747.

13. Lundberg IE, Tjärnlund A, Bottai M, et al. European League Against Rheumatism/American College of Rheumatology classification criteria for adult and juvenile idiopathic inflammatory myopathies and their major subgroups. Arthritis Rheumatol. 2017; 69: 2271-2282.

14. Audet GN, Fulks D, Stricker JC, Olfert IM. Chronic delivery of a thrombospondin-1 mimetic decreases skeletal muscle capillarity in mice. PLoS One. 2013; 8: e55953.

15. Salajegheh M, Raju R, Schmidt J, Dalakas MC.
Upregulation of thrombospondin-1(TSP-1) and its binding partners, CD36 and CD47, in sporadic inclusion body myositis. J Neuroimmunol. 2007; 187: 166-174.

16. Phelan MW, Forman LW, Perrine SR, Faller DV. Hypoxia increases thrombospondin-1 transcript and protein in cultured endothelial cells. J Lab Clin Med. 1998; 132: 519-529.

17. Mansfield PJ, Suchard SJ. Thrombospondin promotes chemotaxis and haptotaxis of human peripheral blood monocytes. J Immunol. 1994; 153: 4219-4229.

18. Le Quintrec JS, Le Quintrec JL. Drug-induced myopathies. Baillieres Clin Rheumatol. 1991; 5: 21-38

19. Aydintug AO, Cervera R, D'Cruz D, et al. Polymyositis complicating D-penicillamine treatment. Postgrad Med. J. 1991; 67: 1018-1020.

20. Preedy VR, Marway JS, Baldwin D, et al. Experimental D-penicillamine-induced myopathy. Biochem Soc Trans. 1996; 24: 2403.

21. Sato N, Okamoto S, Mori T, et al. Recurrent acute myositis after allogeneic bone marrow transplantation for myelodysplasia. Hematology. 2002; 7: 109-112.

22. Oda K, Nakaseko C, Ozawa S, et al. Fasciitis and myositis: An analysis of muscle-related complications caused by chronic GVHD after allo-SCT. Bone Marrow Transplant. 2009; 43: 159-167.

23. Hedermann G, Marquart H, Vissing J. Polymyositis following autologous haematopoietic stem cell transplantation. Scand J Rheumatol. 2016; 45: 429-231.

24. Radke J, Pehl D, Aronica E, et al. The lymphoid follicle variant of dermatomyositis. Neurol Neuroimmunol Neuroinflammation. 2014; 1: e19.

25. De Padilla CML, Vallejo AN, Lacomis D, et al. Extranodal lymphoid microstructures in inflamed muscle and disease severity of new-onset Juvenile dermatomyositis. Arthritis Rheum. 2009; 60: 1160-1172.

26. Radke J, Koll R, Preuße C, et al. Architectural B-cell organization in skeletal muscle identifies subtypes of dermatomyositis. Neurol Neuroimmunol Neuroinflammation. 2018; 5: e451.

27. Jung M, Bonner A, Hudson M, et al. Canadian Scheroderma Research Group (CSRG). Myopathy is a poor prognostic feature in systemic sclerosis: results from the Canadian Scleroderma Research Group (CSRG) cohort. Scand J Rheumatol. 2014; 43: 217-220.

28. Chaigne B, Rodeia S, Benmostefa N, et al. Corticosteroid-sparing benefit of intravenous immunoglobulin in systemic sclerosis-associated myopathy: A comparative study in 52 patients. Autoimmun Rev. 2020; 19: 102431.

29. Ranque B, Authier FJ, Le-Guern V, et al. A descriptive and prognostic study of systemic sclerosis-associated myopathies. Ann Rheum Dis. 2009; 68: 1474-1477. 